\title{
Subthalamic nucleus lesions increase impulsive action and decrease impulsive choice - mediation by enhanced incentive motivation?
}

\author{
Jason M. Uslaner* and Terry E. Robinson \\ Biopsychology and Neuroscience Programs, Department of Psychology, University of Michigan, East Hall, 525 E. University Street, \\ Ann Arbor, MI 48019-1109, USA
}

Keywords: amphetamine, attention deficit hyperactivity disorder, basal ganglia, delay discounting, differential reinforcement of low rates of responding, rat

\begin{abstract}
The subthalamic nucleus (STN) is traditionally thought of as part of a system involved in motor control but recent evidence suggests that it may also play a role in other psychological processes. Here we examined the effects of STN lesions on two measures of impulsivity and found that STN lesions increased 'impulsive action' (produced behavioral disinhibition), as measured by performance on a differential reinforcement of low rates of responding task, but decreased 'impulsive choice' (impulsive decision making), as measured by a delay discounting task. In addition, amphetamine and food restriction increased 'impulsive action' and decreased 'impulsive choice' to a greater extent in STN-lesioned animals than in sham controls. We speculate that these apparently discrepant effects may be because STN lesions enhance the incentive salience assigned to rewards. These findings suggest that the STN may serve as a novel target for the treatment of psychological disorders characterized by deficits in behavioral control, such as drug addiction and attention deficit hyperactivity disorder.
\end{abstract}

\section{Introduction}

The subthalamic nucleus (STN) is traditionally considered a structure involved in motor control (Albin et al., 1989; Crossman, 1990) but recent evidence suggests that it is also involved in mediating the behavioral effects of drugs of abuse and in impulse control. For example, bilateral lesions of the STN increase the behavioral and reinforcing effects of cocaine, as measured by cocaine-induced psychomotor activation, the acquisition of cocaine self-administration and performance on a progressive ratio schedule (Uslaner et al., 2005; but see Baunez et al., 2005). A role for the STN in impulse control is suggested by experiments showing that STN lesions increase impulsive behavior, as indicated by premature anticipatory responding to a cue signaling reward (Baunez et al., 1995; Baunez \& Robbins, 1997, 1999; Phillips \& Brown, 1999; Florio et al., 2001).

Although most studies report that STN lesions increase impulsivity, Winstanley et al. (2005) recently reported that STN lesions decrease impulsivity. They speculated that this apparent discrepancy might be because they studied a different subcomponent of impulsivity than in other studies. Specifically, Winstanley et al. (2005) employed a delay discounting task, in which an animal must choose between a small immediate reward vs. a large reward at a specified delay. They found that STN lesions increased the propensity to choose the larger delayed

Correspondence: Dr Jason M. Uslaner, at *present address below.

E-mail: Jason_Uslaner@merck.com

*Present address: Department of Biology, Merck Research Laboratories, P.O. Box 4, West Point, PA, 19486.

Received 7 June 2006, revised 27 July 2006, accepted 16 August 2006 reward. They hypothesized that STN lesions may increase 'impulsive action' (or decrease behavioral inhibition), as indicated by the premature responding effects reported by others (Baunez et al., 1995; Baunez \& Robbins, 1997, 1999; Phillips \& Brown, 1999; Florio et al., 2001), but decrease 'impulsive choice', or impulsive decision making, as indicated by their results using the delay discounting task.

The purpose of the present experiment was threefold. First, we sought to test the hypothesis proposed by Winstanley et al. (2005) by directly comparing the effects of STN lesions on the following.

(i) Performance on a task involving the differential reinforcement of low rates of responding (DRL)-30-s. This task is specifically designed to measure 'impulsive action' by requiring animals to wait at least $30 \mathrm{~s}$ between responses in order to receive reward. This task causes animals to slow down their response rate and high rates of responding are indicative of 'impulsive action'.

(ii) A delay discounting task, which is designed to measure 'impulsive choice' (Evenden, 1999).

Second, we aimed to further characterize the influence of STN lesions on the effects of psychostimulant drugs by examining the ability of amphetamine to modulate performance on both the DRL-30-s and delay discounting tasks in animals with STN lesions. We predicted that STN lesions would potentiate the effects of amphetamine on both tasks. Third, we examined the influence of food restriction on impulsive responding in animals with STN lesions. We predicted that STN lesions would increase the ability of food restriction to influence impulsivity, indicating that STN-lesioned animals are more sensitive to increases in incentive motivation resulting from both psychostimulant administration and food restriction. 


\section{Materials and methods}

\section{Experiment 1. Influence of bilateral subthalamic nucleus lesions} on differential reinforcement of low rates of responding

\section{Subjects}

Thirty-nine male Sprague-Dawley rats (Harlan, Indianapolis, IN, USA) weighing 225-250 g were housed individually in clear square plastic cages and were given a 1-week acclimatization period before any experimental manipulation. The rooms were temperature and humidity controlled, and maintained on a 14/10 h light/dark cycle (lights on at 07:00 h), with food and water available ad libitum. All experimental procedures were approved by the University of Michigan Committee on the Use and Care of Animals.

\section{Apparatus}

Behavioral testing was conducted in standard operant chambers (Medical Associates, Inc., Georgia, VT, USA) containing an acrylic hinged loading door, stainless steel side panels and an acrylic back panel $(22 \times 18 \times 13 \mathrm{~cm})$. The chambers were located in sound- and light-attenuating cabinets equipped with fans providing constant ventilation and low-level background noise, and a house light providing illumination. Centered on one wall of the chamber was a food cup into which $45 \mathrm{mg}$ banana-flavored pellets could be delivered. To the right or left of the food cup (counterbalanced across animals) was a lever that remained extended throughout the session.

\section{Training procedures}

Animals were first trained on a fixed ratio 1 schedule of reinforcement to lever press for banana-flavored pellets. Sessions lasted $45 \mathrm{~min}$ and were conducted for three consecutive days. Animals were then trained on a DRL-5-s schedule in which a lever press resulted in pellet delivery only if at least $5 \mathrm{~s}$ had elapsed from the previous press (other than for the first response). Following 5 days of training, the schedule was changed to DRL-10-s, 5 days later to DRL-20-s and 5 days later to DRL-30-s. Animals performed on the DRL-30-s schedule for 15 days, after which they underwent surgery (see below). Following surgery and 12-14 days recovery, animals were reintroduced to the DRL-30-s schedule for 10 days to examine the influence of bilateral STN lesions on performance. Then, in order to assess the influence of food restriction, animals were restricted to $16 \mathrm{~g}$ chow per day in their home cage for 3 days and reintroduced to the DRL-30-s schedule. Animals were maintained on $16 \mathrm{~g}$ /day for four more days and continued to perform the task each day (being fed $1 \mathrm{~h}$ after task completion). Animals were then again given free access to chow in their home cages for 3 days before being reintroduced to the task for three more days. Finally, in order to examine the effects of amphetamine, animals were given an injection of $0,0.375$ or $0.75 \mathrm{mg} / \mathrm{kg}$ D-amphetamine (i.p.) $3 \mathrm{~min}$ before being placed in the operant box to perform the task. A single dose of amphetamine was administered to each rat on three separate consecutive test days such that each animal received all three doses, and a Latin squares design was used to counterbalance and control for any order effects.

\section{Surgical procedures}

Surgical procedures were identical to those described in Uslaner et al. (2005). Rats were anesthetized with ketamine hydrochloride (75 mg/kg i.p.) (Fort Dodge Animal Health, Fort Dodge, IA, USA) and xylazine hydrochloride (7.5 mg/kg i.p.) (Ben Venue Laboratories, Bedford, OH, USA) and secured into a stereotaxic apparatus. Twentytwo rats received bilateral injections of ibotenic acid $[12.5 \mu \mathrm{g} / 1$ in $0.1 \mathrm{M}$ phosphate-buffered saline ( $\mathrm{pH} \mathrm{7.4);} \mathrm{Tocris} \mathrm{Cookson} \mathrm{Inc.,}$
Ellisville, MO, USA] and the remaining 17 rats received vehicle alone $(0.1 \mathrm{M}$ phosphate-buffered saline). The two groups were matched for pre-operative performance. The coordinates targeting the STN were measured from bregma: anterior/posterior, $-3.8 \mathrm{~mm}$; lateral, $+2.4 \mathrm{~mm}$; dorsal/ventral, $-8.35 \mathrm{~mm}$ (from skull). The volume injected was $0.5 \mu \mathrm{L}$ per side infused over 3 min using a $10-\mu \mathrm{L}$ Hamilton syringe connected by tubing fitted to a 30-gauge stainless steel injector needle. A micropump was calibrated to deliver the exact volume of fluid.

\section{Verification of lesion}

After the completion of testing, animals were deeply anesthetized with isoflurane, decapitated and their brains were removed, frozen in isopentane on dry ice, and stored at $-70{ }^{\circ} \mathrm{C}$. Brains were sectioned using a cryostat and $16-\mu \mathrm{m}$ coronal sections were thaw-mounted to Superfrost/Plus slides (Fisher Scientific, Pittsburgh, PA, USA) and stained with cresyl violet to assess the extent of the lesions.

\section{Statistics}

The total number of responses (lever presses) was used as an indicator of 'impulsive action', with greater responding indicating higher levels of behavioral disinhibition. In addition, the number of reinforcers earned per session was analysed. Furthermore, in order to characterize changes in the pattern of responding resulting from STN lesions, peak deviation analysis was used to examine the distribution of interresponse times (IRTs) (Richards \& Seiden, 1991; Richards et al., 1993). Briefly, the aim of peak deviation analysis is to compare the IRT distribution achieved by an animal with the IRT distribution that would have been achieved had the animal randomly emitted the same number of responses over the same period of time (characterized by the corresponding negative exponential distribution). Three measurements result from peak deviation analysis: burst ratio, peak area and peak location (Richards \& Seiden, 1991; Richards et al., 1993). The burst ratio is the number of obtained burst responses (the number of responses occurring within $3 \mathrm{~s}$ of a previous response) divided by the number of burst responses predicted by the corresponding negative exponential. Thus, an increase in burst ratio is an indicator of 'impulsive actions' that are perseverative in nature. To determine peak area and peak location, a peak-finding algorithm was used to locate the largest deviation of the obtained pause distribution above the corresponding negative exponential (pause responses are those occurring $>3 \mathrm{~s}$ from a previous response). Thus, the peak represents pause responses with IRTs that occurred at greater than chance levels. Peak location is defined as the median IRT duration of the peak, whereas peak area is defined as the proportion of the IRT durations in the peak. A decrease in peak location indicates that peak responding occurred at a shorter IRT. In contrast, a decrease in peak area indicates that the proportion of responses within the peak has decreased, such that a smaller proportion of responses are above that predicted by random responding. Peak location and peak area are dissociable (Richards \& Seiden, 1991; Richards et al., 1993) but a decrease in either measure is indicative of a pattern of responding that is impulsive.

The total number of responses, reinforcers, burst ratio, peak area and peak location were analysed using a mixed-model ANOVA with session and lesion included in the model. Mixed-model ANOVA is especially appropriate for analysing data with repeated measures, when correlations among the measurements are likely, and allows for greater flexibility in modelling time effects than other repeatedmeasures analyses (Gueorguieva \& Krystal, 2004). A Satterthwaite approximation for the denominator degrees of freedom was used, 
producing decimal places in these values. Mixed-model ANOvA was also used to examine whether food restriction differentially influenced DRL performance in STN-lesioned animals by including session and lesion in the model (data from the session before and after food restriction plus the five sessions in which animals were food restricted were included). Finally, to examine the effect of amphetamine on DRL performance, dose and lesion were included in the model. Significant interactions were followed by independent $t$-tests and the modified Bonferroni procedure was used to control for multiple comparisons. The modified Bonferroni approach maintains Type I error rates at the 0.05 level and has greater power than the traditional Bonferroni procedure (Holland \& Copenhaver, 1988). Uncorrected $P$-values were compared with corrected $\alpha$-values.

\section{Experiment 2. Influence of bilateral subthalamic nucleus lesions on delay discounting}

\section{Subjects}

Thirty-nine male Sprague-Dawley rats (Harlan) weighing 225-250 g were housed individually in clear square plastic cages and were given a 1-week acclimatization period before any experimental manipulation. The rooms were temperature and humidity controlled and on a 14/10 h light/dark cycle (lights on at 07:00 h). Water was available ad libitum, whereas food was available ad libitum for the first 5 days of acclimatization, after which the animals were maintained on $18 \mathrm{~g}$ chow per day in order to keep their body weight at $90 \%$ of their freefeeding weight (we have found that food restriction ensures that animals consume all of their pellets during testing).

\section{Apparatus}

Behavioral testing was conducted in the same operant chambers as in Experiment 1. Centered on one wall of the chamber was a food cup into which $45 \mathrm{mg}$ banana-flavored pellets could be delivered. A stimulus light (tray light) was positioned above the food cup. In addition, two levers that could be extended and retracted were positioned to the left and right of the food cup. When extended, the levers were approximately $3 \mathrm{~cm}$ above the floor. There was also a stimulus light located behind each lever (lever light), which illuminated the lever only when it was extended.

\section{Training procedures}

The training and testing procedures were adapted from Evenden \& Ryan (1996) and Cardinal et al. (2000). On the first day of training animals were brought to the operant chambers and given banana pellets on a random interval schedule with a mean intertrial interval of $30 \mathrm{~s}$ in order to familiarize the animals with retrieving the pellets from the food cup. This session lasted $25 \mathrm{~min}$. Next, animals were trained on an fixed ratio 1 schedule of reinforcement to lever press for banana pellets, first with the lever on one side of the food cup extended throughout the session and then with the lever on the other side (counterbalanced right and left). Animals continued training on the fixed ratio 1 schedule until they reached a criterion of 50 presses in $30 \mathrm{~min}$ for both levers.

Animals were then trained on a simplified version of the full task. Sessions consisted of 90 trials, each of which lasted $40 \mathrm{~s}$. The session began with the house light off and the levers retracted. At the onset of each trial, the house light and tray light were activated and the animal was required to poke its nose into the food cup within $10 \mathrm{~s}$ or the current trial was aborted and the house light and tray light were turned off. If the animal did nose poke within the $10 \mathrm{~s}$, the tray light was turned off and a lever (either the right or left; randomly selected) was extended and the lever light was activated. If the animal failed to make a lever press within $10 \mathrm{~s}$ the current trial was aborted, the lever was retracted, and the house light and lever light were turned off. If the animal did lever press, the lever was retracted, the lever light was turned off, a single pellet was delivered into the food cup and the tray light was turned on. Once the animal made a nose poke into the food cup or $10 \mathrm{~s}$ elapsed the tray light was turned off and the house light was turned off $6 \mathrm{~s}$ later. Animals were trained to a criterion of 60 successful trials (90 being the maximum possible).

\section{Testing procedures}

Test sessions consisted of 60 trials, each trial lasting $100 \mathrm{~s}$. They began with the house light off and the levers retracted. At the onset of each trial, the house light and tray light were activated and the animal was required to poke its nose into the food cup within $10 \mathrm{~s}$ or the current trial was aborted and the house light and tray light were turned off until the next trial. If the animal did nose poke, the tray light was turned off and either one or both levers were extended (one lever for forced trials and both levers for choice trials). One lever was designated as the immediate lever and the other as the delay lever (right-left counterbalanced across animals). If the animal failed to press a lever within $10 \mathrm{~s}$ the current trial was aborted, the house light was turned off and the levers were retracted. If the animal pressed the immediate lever, the levers were retracted, the tray light was activated and one pellet was immediately delivered into the food cup. If the animal pressed the delay lever, the extended levers were retracted, the tray light was activated and four pellets were delivered into the food cup at a specified delay (see below). After the animal made a nose poke into the food cup or $10 \mathrm{~s}$ elapsed the tray light was turned off and the house light was turned off $6 \mathrm{~s}$ later.

Each session consisted of five blocks of 12 trials. For each block, the first six trials were forced choice trials, in which only the immediate or delay lever was extended (three immediate and three delay lever presentations pseudorandomly selected). Forced trials were included to encourage the animal to sample from both levers and also to decrease any side bias. The last six trials of each block were choice trials in which both levers were extended allowing the animal to choose between the immediate and delay levers. The delay associated with the delay lever was systematically varied across trial blocks. For the first eight sessions, the delay associated with each block of trials was $0,6,12,24$ and $48 \mathrm{~s}$. However, because animals almost always chose the immediate lever at both the 24- and 48-s delay (such that including both delays was redundant), the delays were changed from session 9 onward to $0,3,6,12$ and $24 \mathrm{~s}$.

After 24 sessions animals were divided into groups matched on performance, underwent surgery (see below), allowed to recover for 12-14 days and reintroduced to the task to examine the influence of STN lesions on performance. Following 8 days of testing, the influence of amphetamine on test performance as a function of STN lesion was examined over 3 days by administering (i.p.) $0,0.15$ or $0.3 \mathrm{mg} / \mathrm{kg}$ D-amphetamine $3 \mathrm{~min}$ before the task started. A single dose of amphetamine was administered to each rat on three separate consecutive test days such that each animal received all three doses, and a Latin squares design was used to counterbalance and control for possible order effects.

\section{Surgical procedures and verification of the lesion}

Surgeries and lesion verification were conducted in the same way as in Experiment 1 (see above). Twenty-two animals received bilateral infusions of ibotenic acid aimed at the STN and 17 animals received vehicle alone. 


\section{Statistics}

Lever press data for each block were converted into percentages in which animals chose the delayed reward by dividing the number of presses on the delay lever by the total number of lever presses for that block (maximum 6) and multiplying by 100 . Then, in order to examine if bilateral STN lesions influenced choice between the immediate vs. delayed reward, mixed-model ANOVA was used with lesion and delay included in the model. Significant interactions were followed by $t$-tests to examine the duration of delay for which significant differences occurred between STN-lesioned animals and sham controls. The influence of amphetamine as a function of STN lesion was also examined with mixed-model ANOVA. First, to examine if amphetamine produced an effect in controls and/or STNlesioned animals, dose and delay were included in the model and mixed-model ANOVA was performed separately for the control and lesion groups. Then, in order to examine if amphetamine differentially influenced controls and STN-lesioned animals, mixed-model ANOVA was performed with dose, lesion, and delay included in the model.

\section{Results}

\section{Experiment 1. Differential reinforcement of low rates of responding}

\section{Histology}

The size of the lesion was verified by examining cresyl violet-stained sections to assess the extent of cell loss and gliosis in the STN and surrounding areas. Of the 22 rats injected with ibotenic acid, 17 showed extensive bilateral damage to the STN. Three animals showed cell loss unilaterally and two animals did not have detectable cell loss. These latter five animals were not included in the data analysis. Figure 1 shows photomicrographs of representative cresyl violetstained sections, illustrating the extent of the lesion. Cell loss was mainly restricted to the STN, with little damage extending dorsally or medially into the zona incerta.

\section{Effect of subthalamic nucleus lesions}

The left column of graphs in Fig. 2 shows the effects of STN lesions on different measures of DRL-30-s schedule performance as a function of test session. Mixed-model ANOVA revealed that STN lesions increased both the total number of responses and burst ratio, and decreased peak area (main effect of day and day $\times$ lesion interaction; all $P$-values $<0.03$ ). Thus, STN lesions increased 'impulsive action' as revealed by the greater amount of total responding, and also influenced the pattern of responding, increasing responses with short IRTs (burst ratio) and decreasing the number of responses within the peak (i.e. responding was closer to random), relative to controls.

Although the effect of STN lesions on total responding decreased with repeated testing, there was a long-lasting increase in burst ratio and decrease in peak area (all post-lesion sessions $P$-values significant at the modified Bonferroni $\alpha$-value, other than burst ratio for sessions 4 and 6 , whereas the effect of the lesion on total responses was significant on test days 1 and 2 post-lesion $P$-values $<0.005$ but not thereafter).

Finally, in contrast to effects on total responses, burst ratio and peak area, STN lesions had no influence on the total number of reinforcers earned or peak location. The absence of an effect on the number of reinforcers earned was probably due to the control group losing greater amounts of time for each premature response (i.e. premature responses closer to the 30 -s time point), whereas lesioned animals lost less time for each premature response (more of their premature responses were burst responses). The finding that peak location was the same for both groups indicates that the IRT of peak responding was the same for both groups, even though the number of responses in the peak was smaller for the lesion group, as indicated by the decrease in peak area. Thus, STN-lesioned animals did show a level of responding that peaked at the same IRT as controls but showed less responding within the peak. This pattern of deficits indicates that STN-lesioned animals do not lack the ability to distinguish time (as revealed by no change in peak location) despite showing impulsive responding (as indicated by an increase in total responses; see Discussion for further comments on this point).

To further illustrate the effects of STN lesions, we graphed the raw IRTs plotted in 1-s bins for the session before the lesion (Fig. 3A), the first day of testing after surgery (Fig. 3B) and the 10th day of testing after surgery (Fig. 3C). The number of responses with IRTs $>40 \mathrm{~s}$ was very small and is not included in order to ease representation. It is clear from Fig. 3 that the pattern of responding is very different for STN-lesioned animals relative to shams, with STN lesions increasing total responses on the first day of testing and producing a persistent increase in burst responding and a decrease in the size of the peak on both the first and 10th day, supporting the statistical results from peak deviation analysis described above.
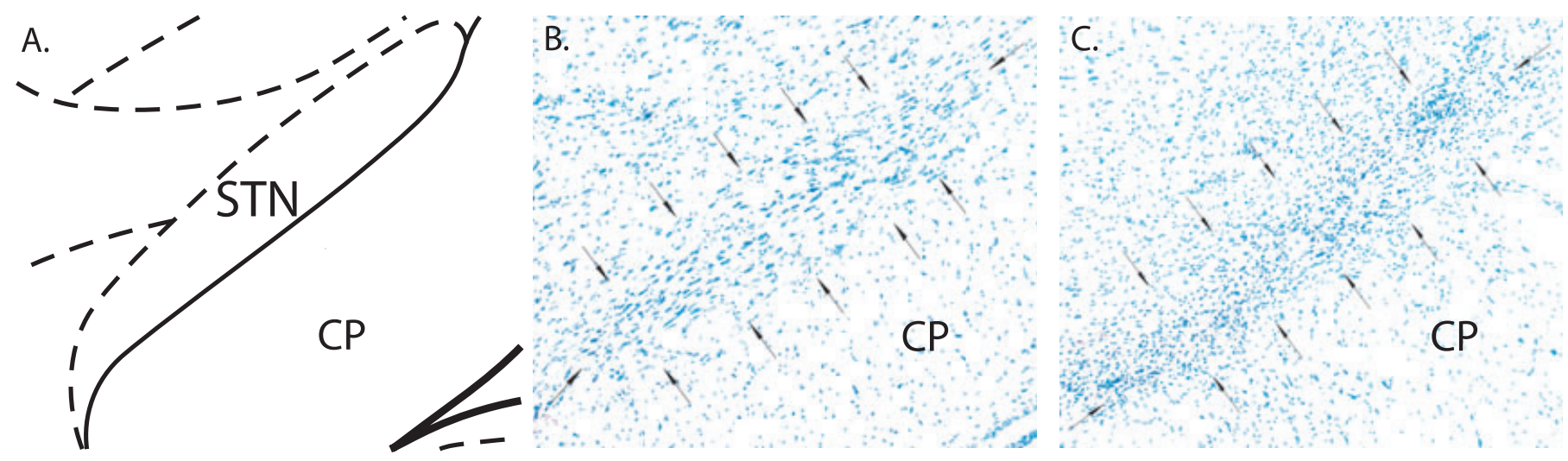

FIG. 1. Illustration showing the subthalamic nucleus (STN) and bordering cerebral peduncle (CP). (A) Representative cresyl violet-stained sections at the level of the subthalamic nucleus (STN) in a sham control (B) and in an animal with an STN lesion (C). Arrows indicate the boundaries of the STN. Gliosis and cell loss are apparent in the cresyl violet-stained section of the lesioned animal. The cerebral peduncle (CP) is labeled to aid anatomical location. 


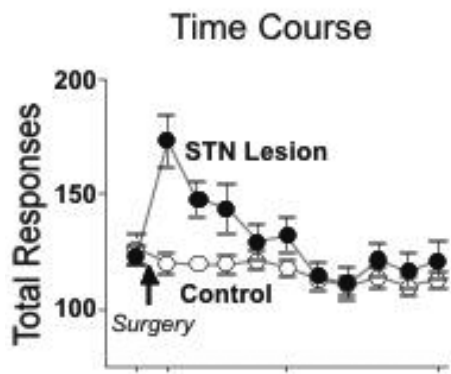

Food Restriction
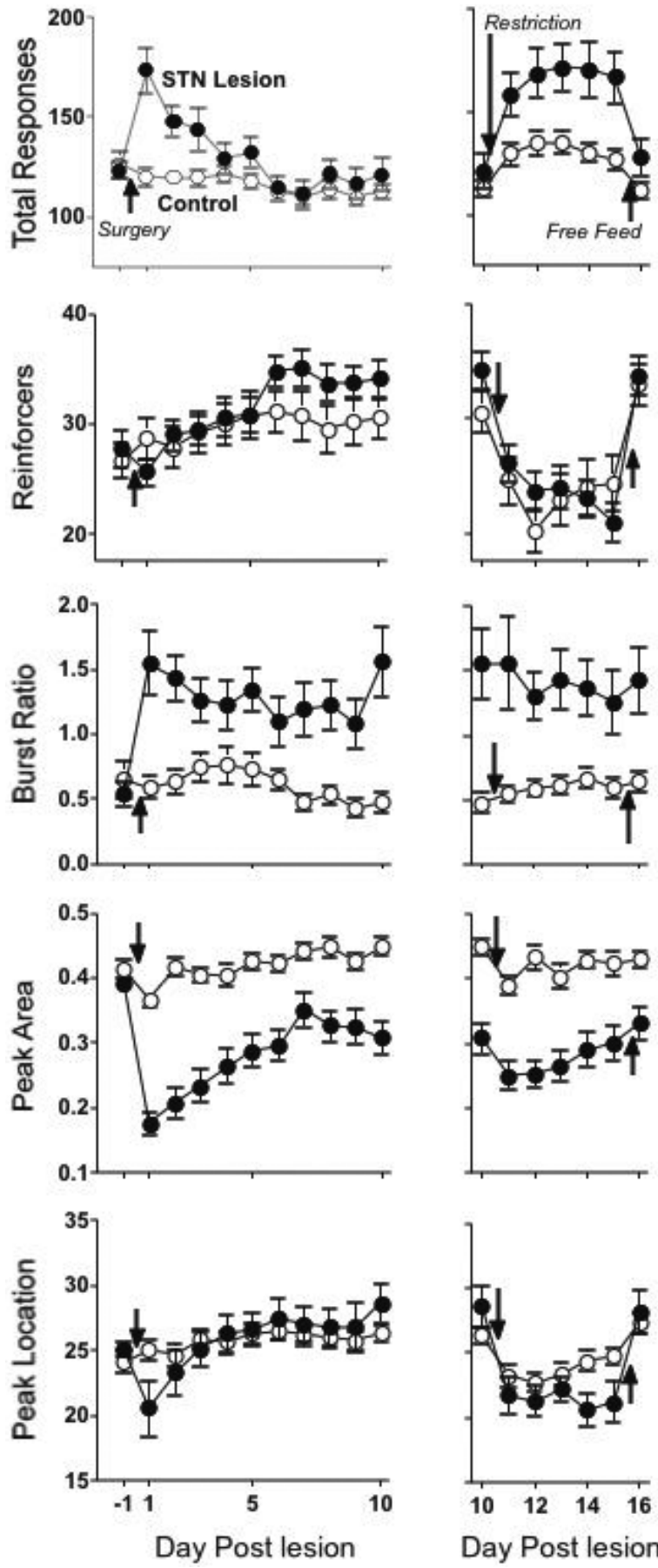
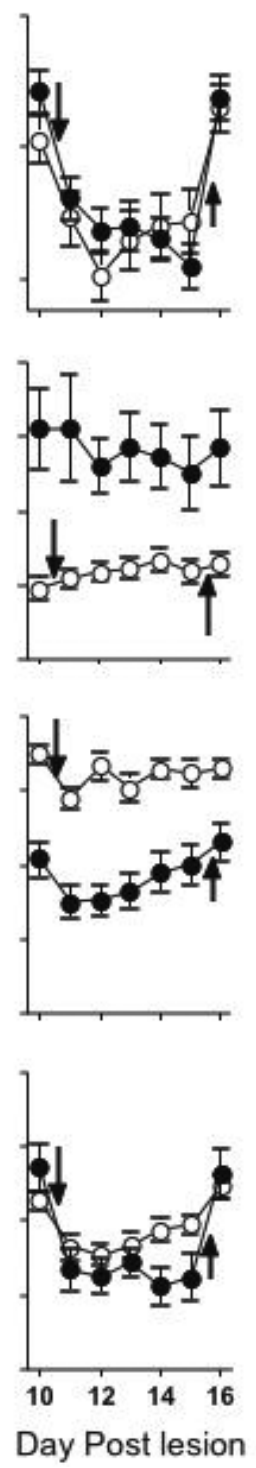

Amphetamine
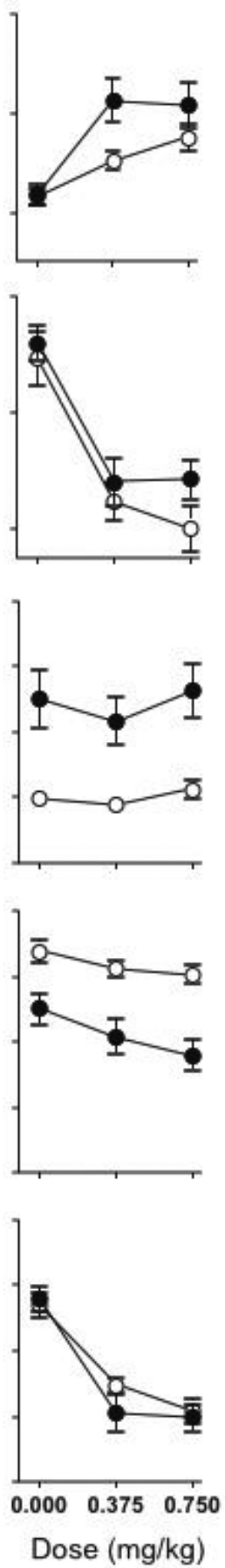

FIG. 2. Measurements of performance on a differential reinforcement of low rates of responding-30-s schedule in subthalamic nucleus (STN)-lesioned and control animals as a function of test session, food restriction and amphetamine treatment. Data points represent mean \pm SEM. $\bullet$, STN-lesioned animals; $\bigcirc$, sham controls.

\section{Effect of food restriction}

The middle column of graphs in Fig. 2 shows performance on the DRL-30-s schedule as a function of food restriction. Food restriction increased the total number of responses and decreased the number of reinforcers earned, peak area, and peak location (main effect of day; all $P$-values $<0.001)$. Importantly, food restriction enhanced total responding to a greater extent in STN-lesioned animals relative to controls (day $\times$ lesion interaction; $F_{6,190.59}=3.28 ; P=0.004$ ). Spe- cifically, the total number of responses made by controls and animals with STN lesions did not differ on the session before or after food restriction but STN-lesioned animals responded more on all 5 days in which they were restricted (all $P$-values $<0.01$ ). In addition, peak location was also decreased by food restriction to a greater extent in STN-lesioned animals (day $\times$ lesion interaction; $F_{6,164.47}=3.12$; $P=0.006)$. There was no difference in peak location between animals with STN lesions and controls on the session before or after food restriction but there was a trend for a greater food-restriction- 

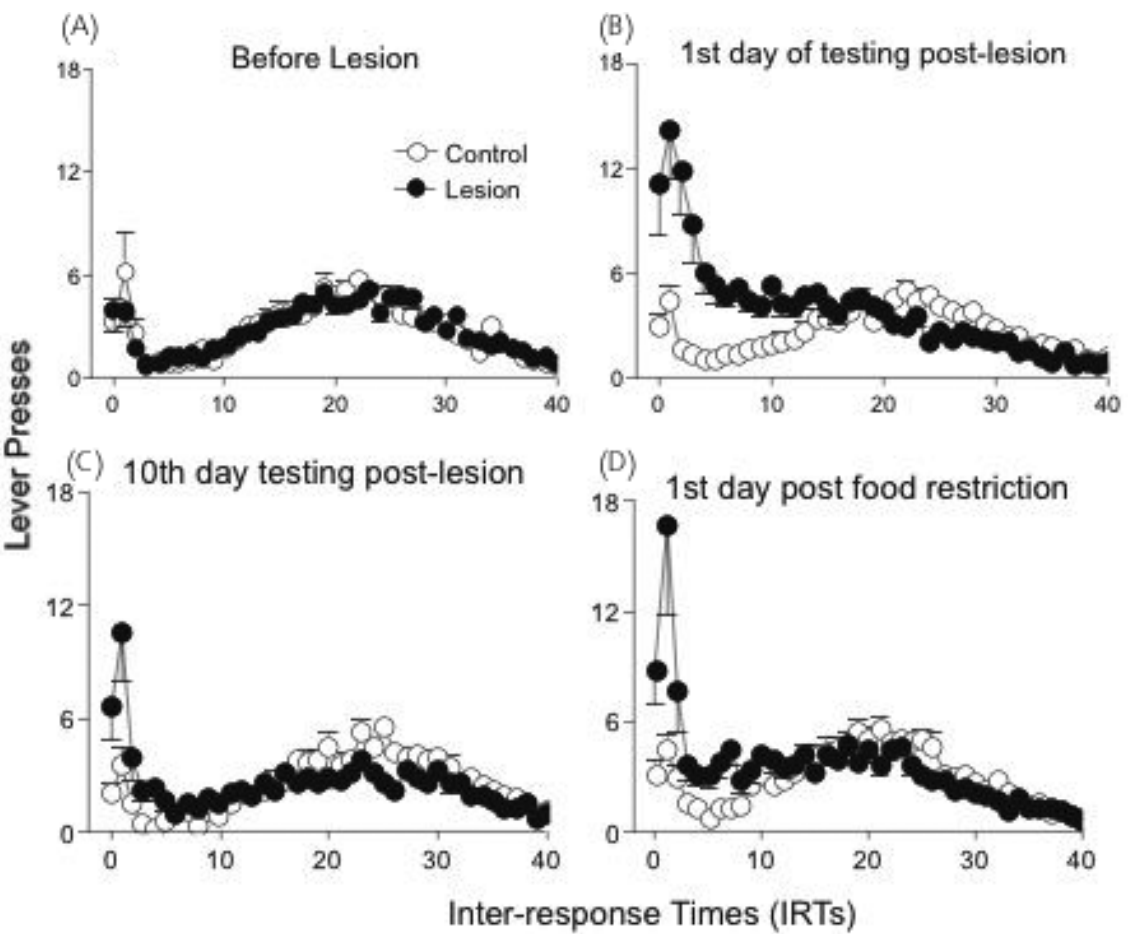

FIG. 3. Raw inter-response times plotted in 1-s bins for the differential reinforcement of low rates of responding-30-s session before the lesion (A), the first day of testing after surgery (B), the 10th day of testing after surgery $(\mathrm{C})$ and the first test day after food restriction (D). Data points represent mean \pm SEM.

induced decrease in STN-lesioned animals, relative to controls, on days 14 and 15 (days 4 and 5 of food restriction; $P$-values $=0.021$ and 0.05 , respectively; nonsignificant according to modified Bonferroni). The effect of food restriction on all other measurements did not vary as a function of lesion. In summary, food restriction increased the total number of responses to a greater extent in STN-lesioned animals relative to controls, indicating a greater increase in 'impulsive action', and changed the pattern of responding by decreasing the median IRT of the peak to a greater extent in STN-lesioned animals.

Figure 3D further illustrates the effect of food deprivation in STNlesioned animals by showing the IRTs plotted in 1-s bins for the first day of food restriction. By comparing the IRT distributions of the day prior to food restriction (Fig. 3C) with the first day after food restriction, it is apparent that the number of responses and location of the peak were influenced by an interaction between STN lesion and food restriction, consistent with the statistical results from peak deviation analysis described above.

\section{Effect of amphetamine}

The right column of graphs in Fig. 2 shows performance on the DRL30 -s task as a function of amphetamine treatment. Amphetamine dosedependently increased the total number of responses and decreased the number of reinforcers earned, peak area, and peak location in both groups (main effect of dose; all $P$-values $<0.013$ ). Importantly, the dose-effect curve for the ability of amphetamine to increase total responses was shifted to the left in STN-lesioned animals, relative to controls (dose $\times$ lesion interaction; $F_{2,67.04}=4.68 ; P=0.013$ ). Thus, at the lowest dose of amphetamine tested $(0.375 \mathrm{mg} / \mathrm{kg})$, amphetamine produced a significantly greater response in animals with STN lesions, as compared with controls $\left(t_{33}=2.44 ; P=0.015\right)$. In contrast, there was no difference between controls and STN-lesioned animals at $0.75 \mathrm{mg} / \mathrm{kg}$ amphetamine. Furthermore, the ability of amphetamine to increase total responding in lesioned animals, relative to controls, was not marked by a change in the pattern of IRT distribution, as revealed by nonsignificant interactions between dose and lesion for burst ratio, peak area, and peak location. Nevertheless, the effect on total responses is consistent with the idea that amphetamine increases 'impulsive action' to a greater extent in STN-lesioned animals, relative to controls.

\section{Experiment 2. Delay discounting}

\section{Histology}

The size of the lesion was verified in the same manner as in Experiment 1. Of the 22 rats injected with ibotenic acid, 18 showed extensive bilateral damage to the STN, with little or no apparent damage outside the structure. Three animals showed cell loss unilaterally and one animal had no detectable damage. These latter four animals were excluded from the analysis.

\section{Effect of subthalamic nucleus lesion}

The leftmost graph in Fig. 4 shows the percentage of responses on the delay lever as a function of delay period for the first test session after surgery. As the delay increased the percentage of responses on the delay lever decreased (main effect of delay; $F_{4,147.9}=45.77$; $P<0.001$ ), indicating that both groups of rats were influenced by the delay (i.e. responses were not random). Although there was no main effect of lesion $\left(F_{1,79.69}=0.39 ; P=0.53\right)$, there was a significant delay $\times$ lesion interaction $\left(F_{4,147.9}=5.4 ; P=0.02\right)$ and $t$-tests revealed that animals with STN lesions did not differ from controls at the shorter delays $(\leq 12 \mathrm{~s})$ but chose the large reward more frequently at the longest delay $\left(t_{32}=2.16, P=0.038\right)$. This is consistent with the notion that STN lesions decrease 'impulsive choice'. With continued testing this effect of STN lesions abated (data not shown) but, as described below, the effect of STN lesions was 


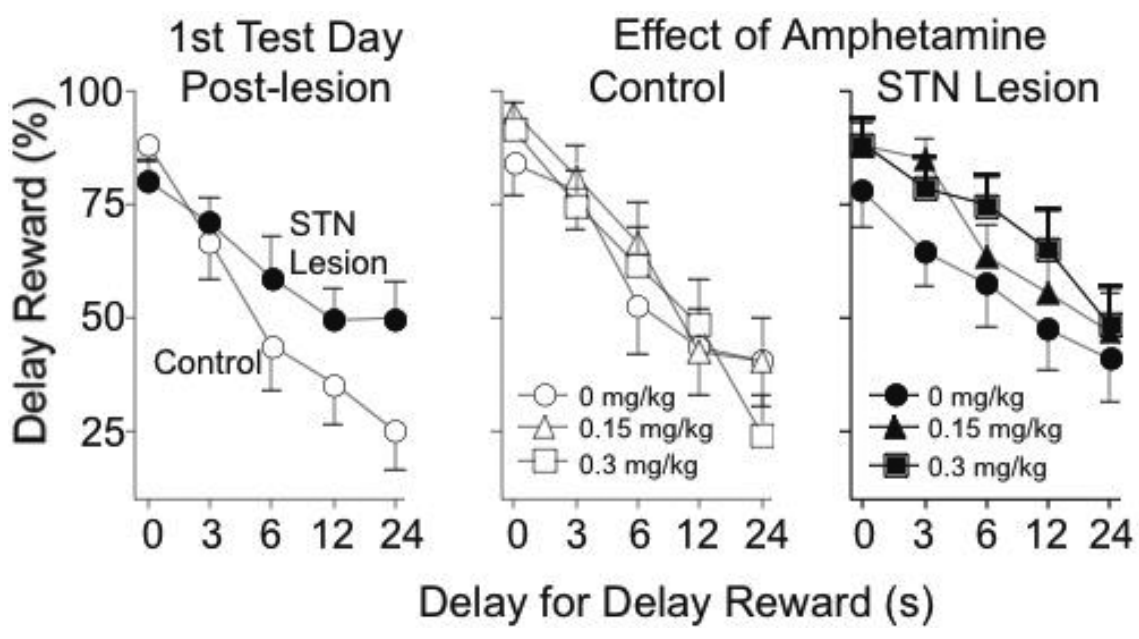

FIG. 4. Performance on the delay discounting task on the first session after subthalamic nucleus (STN) lesion surgery, and the effect of amphetamine on task performance on sham controls and STN-lesioned animals. Data points represent mean \pm SEM.

reinstated by amphetamine treatment. Finally, the number of omissions did not differ between STN-lesioned animals and sham controls on any day tested $(P$-values $>0.05)$.

\section{Effect of amphetamine}

The central and rightmost graphs in Fig. 4 show the response to amphetamine in controls and STN-lesioned animals, respectively. Both controls and animals with STN lesions chose the delay lever more frequently at shorter delays (main effect of delay; controls: $F_{4,111.73}=10.48, P<0.001$; lesion: $\left.F_{4,112.7}=5.34, P<0.001\right)$. At the doses tested there was no effect of amphetamine in controls (main effect of dose; $F_{2,154.87}=1.01 ; P=0.37$, delay $\times$ dose interaction; $F_{8,175.8}=0.9 ; P=0.52$ ). In contrast, in animals with bilateral STN lesions, both doses of amphetamine significantly increased the percentage of responses on the delayed lever (main effect of dose; $\left.F_{2,171.64}=7.95 ; P=0.001\right)$ and this effect was independent of the length of the delay (delay $\times$ dose interaction; $F_{8,189.04}=0.56$; $P=0.81)$. Furthermore, there was a trend towards an interaction between dose and lesion (dose $\times$ lesion interaction; $F_{2,328.86}=2.93$; $P=0.055)$. These data are consistent with the idea that amphetamine decreases 'impulsive choice' to a greater extent in STN-lesioned animals, relative to controls.

\section{Discussion}

The goals of the present experiments were (i) to determine the effects of STN lesions on behaviors indicative of 'impulsive action' (or behavioral disinhibition) and 'impulsive choice' (or impulsive decision making), and (ii) to examine the influence of amphetamine and food restriction on 'impulsive action' and 'impulsive choice' in animals with STN lesions. We report four major findings. First, STN lesions increased measures indicative of 'impulsive action' (Baunez et al., 1995; Baunez \& Robbins, 1997, 1999; Phillips \& Brown, 1999; Florio et al., 2001) as revealed by performance on the DRL-30-s schedule. Second, both food restriction and amphetamine increased 'impulsive action' to a greater extent in STN-lesioned animals, relative to controls. Third, STN lesions decreased measures indicative of 'impulsive choice' as revealed by the delay discounting task (Winstanley et al., 2005). Fourth, amphetamine decreased 'impulsive choice' in STN-lesioned animals to a greater extent than in controls.
Taken together, these results show that STN lesions produce opposite effects on behaviors thought to be indicative of 'impulsive action' vs. 'impulsive choice', and that amphetamine and food restriction potentiate the effects of STN lesions. It is also worth noting that, with additional post-lesion training, animals with STN lesions came to perform like sham controls according to some indices of impulsivity, suggestive of some form of behavioral and/or neurobiological compensation. However, this 'recovery of function' was more apparent than real, given that it was reinstated by food restriction or amphetamine treatment.

At first glance, it may seem surprising that STN lesions increase measures of impulsivity on one task but produce opposite effects on another task. However, a close examination of the literature reveals that a variety of manipulations produce opposite effects on behaviors indicative of impulsive action vs. impulsive choice. For example, both amphetamine and food restriction are reported to increase impulsive action as measured by DRL schedules and other similar tasks (Seiden et al., 1979; Lewis \& Dougherty, 1992; Sabol et al., 1995) but to decrease impulsive choice as measured by delay discounting or similar tasks (Bradshaw \& Szabadi, 1992; Wogar et al., 1992; Ho et al., 1997; Cardinal et al., 2000; Wade et al., 2000; Winstanley et al., 2003; van Gaalen et al., 2006a, b but see Evenden \& Ryan, 1996; Cardinal et al., 2000). Lesions, as well as pharmacological manipulations of the serotonergic system, also produce different effects on these two measures of impulsivity (Winstanley et al., 2004a; Talpos et al., 2006), as do lesions of the nucleus accumbens (Cole \& Robbins, 1989; Cardinal et al., 2001, 2002), orbitofrontal (Chudasama et al., 2003; Winstanley et al., 2004b) and cingulate cortices (Cardinal et al., 2001; Christakou et al., 2004). In fact, it appears that a manipulation that produces contrasting effects on behaviors indicative of 'impulsive action' and 'impulsive choice' is more the rule than the exception.

Therefore, an important question is what basic psychological process is influenced by STN lesions to produce opposite effects on these two behavioral tasks? One possibility is simply that there are two separate and dissociable components of impulsivity, 'impulsive action' and 'impulsive choice' (Evenden, 1999), and that they are differentially controlled by the STN. According to this hypothesis, engagement of the intact STN, or a particular subset of neurons within the structure, may serve to decrease impulsive action but increase impulsive choice. Although this possibility is consistent with our data, we believe that it is not the most parsimonious explanation for 
our findings because it invokes two psychological processes, rather than one.

Another possibility is that the STN is involved in timing. Specifically, given the importance of timing in both the DRL and delay discounting task, STN lesions may disrupt an internal pacemaker responsible for the timing of events and therefore disrupt performance on both tasks. Indeed, the basal ganglia, and the STN specifically, are thought to be involved in interval timing (see Buhusi \& Meck, 2005 for review), and timing dysfunctions, specifically a slowing down of subjective time, provide one way to account for deficits in impulse control (Ho et al., 1999). However, a close examination of the entire data set reveals at least two reasons why timing deficits probably do not account for the results reported here. First, if subjective timing was slowed down as a result of STN lesions, one would expect greater 'impulsive-like' responding on both the DRL-30-s schedule and on the delay discounting task, whereas if subjective timing was sped up by the lesions one would expect less 'impulsive-like' responding on both tasks. However, STN lesions increased impulsive responding on the DRL-30-s task but decreased impulsive responding on the delay discounting task. Second, at least some of the deficits produced by STN lesions are not heavily dependent on timing, whereas some of the spared functions are. For example, one of the more pronounced deficits produced by STN lesions on the DRL-30-s schedule was an increase in burst responding, as reflected by an increase in burst ratio. However, an increase in burst responding is unlikely to result from a dysfunction in internal timing because, by definition, a burst response is one that far precedes the 30-s critical time point (Richards et al., 1993). If a timing deficit was the cause of increased burst responding, it would have to be a very severe deficit indeed. If STN lesions did produce a severe timing deficit then a measure such as peak location, which is heavily dependent on timing, should have been dramatically influenced by STN lesions. This was not the case as peak location was relatively unaffected.

Although at this time it is speculative, a third possible explanation for the effects reported here is that STN lesions magnify the incentive value of rewards. As a result, STN lesions increase impulsive responding in the DRL-30-s task by making the reward more 'wanted' and thus responding more difficult to inhibit. In the delay discounting task, however, STN lesions decrease impulsive responding because the incentive salience of the large reward is disproportionately enhanced and therefore it is chosen more frequently. In other words, depending on the specific design of the task, increasing incentive motivation via STN lesions can produce contrasting effects on different measures of impulsivity.

Several lines of evidence support this hypothesis. First, manipulations that increase incentive motivation, such as food restriction and amphetamine treatment (Berridge, 1996; Wyvell \& Berridge, 2000), produce a similar pattern of behavioral effects on the DRL-30-s and delay discounting tasks as we report here for STN lesions [i.e. increase impulsive responding on DRL schedules and other similar tasks that are designed to measure 'impulsive action' (Seiden et al., 1979; Lewis \& Dougherty, 1992; Sabol et al., 1995) but decrease impulsive responding on delay discounting and other similar tasks designed to measure 'impulsive choice' (Bradshaw \& Szabadi, 1992; Wogar et al., 1992; Ho et al., 1997; Cardinal et al., 2000; Wade et al., 2000; Winstanley et al., 2003; van Gaalen et al., 2006a, b but see Evenden \& Ryan, 1996; Cardinal et al., 2000)]. Second, manipulations that decrease incentive motivation, such as systemic administration of dopamine antagonists (Dickinson et al., 2000), produce an opposite set of effects as we report here, decreasing measures of impulsive action and increasing impulsive choice (O’Donnell \& Seiden, 1983; Pollard \& Howard, 1986; Cardinal et al., 2000; Wade et al., 2000; van
Gaalen et al., 2006a,b). Third, we found that STN-lesioned animals were more sensitive to manipulations of incentive motivation (via food restriction and amphetamine treatment) than controls. This would be expected if the threshold for activating the incentive salience system was lowered by STN lesions. Fourth, we have previously shown that STN-lesioned animals are more sensitive to the incentive motivational effects of cocaine (Uslaner et al., 2005) and Baunez et al. (2002) have reported the same for food. Fifth, STN inactivation increases the firing rate of dopamine-containing neurons (Smith \& Grace, 1992) and increases dopamine levels in the striatum (Bruet et al., 2001; Meissner et al., 2001; Lee et al., 2006), both of which are believed to be critical for incentive salience attribution (Dickinson et al., 2000; Wyvell \& Berridge, 2000, 2001). Thus, we hypothesize that STN lesions magnify the incentive value of rewards and, by extension, the intact STN serves to inhibit inappropriate 'wanting' of incentive stimuli.

Indeed, the STN is in a prime neuroanatomical position to perform this function. Specifically, the STN receives direct input from the medial prefrontal cortex and indirect input from the nucleus accumbens via the ventral pallidum (Monakow et al., 1978; Berendse \& Groenewegen, 1991; Maurice et al., 1998a,b), and there is a good deal of evidence indicating that the medial prefrontal cortex is involved in impulse control (Iversen \& Mishkin, 1970; Damasio, 1996; Robbins, 1996) and that the nucleus accumbens and ventral pallidum play important roles in incentive salience attribution (Wyvell \& Berridge, 2000; Tindell et al., 2005). Thus, the STN may serve as a site of convergence for these two types of information, allowing them to be compared and further processed so that an appropriate behavioral response can be made. In fact, the STN has a robust projection onto the entopeduncular nucleus (globus pallidus internal in primates) and substantia nigra pars reticulata (Kita \& Kitai, 1987; Parent \& Hazrati, 1995), and could regulate incentive motivation-related motor activity through these efferents (Sato \& Hikosaka, 2002). Furthermore, via the projection to the substantia nigra pars reticulata, the STN can indirectly regulate activity of dopaminergic neurons (Smith \& Grace, 1992). Of course, other efferents and afferents may also be important.

In conclusion, the current results suggest that STN lesions have opposite effects on measures of impulsive action vs. impulsive choice and we speculate that this may be because STN lesions alter incentive salience attribution. By extension, we propose that the intact STN serves to inhibit incentive salience attribution and inappropriate responding for incentive stimuli. Indeed, there are now reports showing that high-frequency stimulation of the STN in human subjects, which is effective in alleviating motor symptoms associated with Parkinson's disease by inhibiting the STN via depolarization block (Benazzouz et al., 1993, 1995, 2000; Lozano et al., 2002 but see Garcia et al., 2005), can also influence impulsivity, affect and compulsive behavior in humans (Houeto et al., 2002; Funkiewiez et al., 2003; Hershey et al., 2004; Takeshita et al., 2005). For example, one Parkinson's disease patient undergoing high-frequency stimulation was said to have 'increased his daily dose of levodopa, preferentially in a quick acting form...developed devious strategies to increase his stimulation parameters and tried to obtain new medications from physicians with little experience of treating Parkinson's disease' (Houeto et al., 2002, p. 702 but see Witjas et al., 2005). Thus, in patients receiving high-frequency stimulation for the treatment of movement disorders it will be important not only to assess effects on motor control but also on more complex psychological functions. On a more positive note, the current results also suggest that the STN could serve as a novel target for therapies aimed at treating dysfunctions in behavioral control, such as attention deficit hyperactivity disorder, drug addiction and obesity. 


\section{Acknowledgements}

This work was supported by a grant from the National Institute on Drug Abuse to T.E.R. (R37-DA004294). We also wish to thank Pengwei Yang and James Dell'Orco for their technical assistance, Drs Kent Berridge, Joshua Berke and Martin Sarter for helpful comments on the manuscript and Dr Jerry Richards for supplying the program to perform peak deviation analysis.

\section{Abbreviations}

DRL, differential reinforcement of low rates of responding; IRT, inter-response time; STN, subthalamic nucleus.

\section{References}

Albin, R.L., Young, A.B. \& Penney, J.B. (1989) The functional anatomy of basal ganglia disorders. Trends Neurosci., 12, 366-375.

Baunez, C. \& Robbins, T.W. (1997) Bilateral lesions of the subthalamic nucleus induce multiple deficits in an attentional task in rats. Eur. J. Neurosci., 9, 2086-2099.

Baunez, C. \& Robbins, T.W. (1999) Effects of dopamine depletion of the dorsal striatum and further interaction with subthalamic nucleus lesions in an attentional task in the rat. Neuroscience, 92, 1343-1356.

Baunez, C., Nieoullon, A. \& Amalric, M. (1995) In a rat model of parkinsonism, lesions of the subthalamic nucleus reverse increases of reaction time but induce a dramatic premature responding deficit. J. Neurosci., 15, 6531-6541.

Baunez, C., Amalric, M. \& Robbins, T.W. (2002) Enhanced food-related motivation after bilateral lesions of the subthalamic nucleus. J. Neurosci., 22, $562-568$.

Baunez, C., Dias, C., Cador, M. \& Amalric, M. (2005) The subthalamic nucleus exerts opposite control on cocaine and 'natural' rewards. Nat. Neurosci., 8, 484-489.

Benazzouz, A., Gross, C., Feger, J., Boraud, T. \& Bioulac, B. (1993) Reversal of rigidity and improvement in motor performance by subthalamic highfrequency stimulation in MPTP-treated monkeys. Eur. J. Neurosci., 5, 382389.

Benazzouz, A., Piallat, B., Pollak, P. \& Benabid, A.L. (1995) Responses of substantia nigra pars reticulata and globus pallidus complex to high frequency stimulation of the subthalamic nucleus in rats: electrophysiological data. Neurosci. Lett., 189, 77-80.

Benazzouz, A., Gao, D.M., Ni, Z.G., Piallat, B., Bouali-Benazzouz, R. \& Benabid, A.L. (2000) Effect of high-frequency stimulation of the subthalamic nucleus on the neuronal activities of the substantia nigra pars reticulata and ventrolateral nucleus of the thalamus in the rat. Neuroscience, 99, 289-295.

Berendse, H.W. \& Groenewegen, H.J. (1991) The connections of the medial part of the subthalamic nucleus in the rat: evidence for a parallel organization. In Bernardi, G., Carpenter, M.B., Di Chiara, G., Morelli, M. \& Stanzione, P. (Eds), The Basal Ganglia III. Plenum, New York, pp. 89-98.

Berridge, K.C. (1996) Food reward: brain substrates of wanting and liking. Neurosci. Biobehav. Rev., 20, 1-25.

Bradshaw, C.M. \& Szabadi, E. (1992) Choice between delayed reinforcers in a discrete-trials schedule: the effect of deprivation level. Q. J. Exp. Psychol. B, 44, 1-6.

Bruet, N., Windels, F., Bertrand, A., Feuerstein, C., Poupard, A. \& Savasta, M. (2001) High frequency stimulation of the subthalamic nucleus increases the extracellular contents of striatal dopamine in normal and partially dopaminergic denervated rats. J. Neuropathol. Exp. Neurol., 60, 15-24.

Buhusi, C.V. \& Meck, W.H. (2005) What makes us tick? Functional and neural mechanisms of interval timing. Nat. Rev. Neurosci., 6, 755-765.

Cardinal, R.N., Robbins, T.W. \& Everitt, B.J. (2000) The effects of d-amphetamine, chlordiazepoxide, alpha-flupenthixol and behavioural manipulations on choice of signalled and unsignalled delayed reinforcement in rats. Psychopharmacology (Berl.), 152, 362-375.

Cardinal, R.N., Pennicott, D.R., Sugathapala, C.L., Robbins, T.W. \& Everitt, B.J. (2001) Impulsive choice induced in rats by lesions of the nucleus accumbens core. Science, 292, 2499-2501

Cardinal, R.N., Parkinson, J.A., Lachenal, G., Halkerston, K.M., Rudarakanchana, N., Hall, J., Morrison, C.H., Howes, S.R., Robbins, T.W. \& Everitt, B.J. (2002) Effects of selective excitotoxic lesions of the nucleus accumbens core, anterior cingulate cortex, and central nucleus of the amygdala on autoshaping performance in rats. Behav. Neurosci., 116, 553-567.
Christakou, A., Robbins, T.W. \& Everitt, B.J. (2004) Prefrontal cortical-ventral striatal interactions involved in affective modulation of attentional performance: implications for corticostriatal circuit function. J. Neurosci., 24, 773780.

Chudasama, Y., Passetti, F., Rhodes, S.E., Lopian, D., Desai, A. \& Robbins, T.W. (2003) Dissociable aspects of performance on the 5-choice serial reaction time task following lesions of the dorsal anterior cingulate, infralimbic and orbitofrontal cortex in the rat: differential effects on selectivity, impulsivity and compulsivity. Behav. Brain Res., 146, 105119.

Cole, B.J. \& Robbins, T.W. (1989) Effects of 6-hydroxydopamine lesions of the nucleus accumbens septi on performance of a 5-choice serial reaction time task in rats: implications for theories of selective attention and arousal. Behav. Brain Res., 33, 165-179.

Crossman, A.R. (1990) A hypothesis on the pathophysiological mechanisms that underlie levodopa- or dopamine agonist-induced dyskinesia in Parkinson's disease: implications for future strategies in treatment. Mov. Disord., 5, $100-108$.

Damasio, A.R. (1996) The somatic marker hypothesis and the possible functions of the prefrontal cortex. Philos. Trans. R. Soc. Lond. B Biol. Sci., 351, 1413-1420.

Dickinson, A., Smith, J. \& Mirenowicz, J. (2000) Dissociation of Pavlovian and instrumental incentive learning under dopamine antagonists. Behav. Neurosci., 114, 468-483.

Evenden, J.L. (1999) Varieties of impulsivity. Psychopharmacology (Berl.), 146, 348-361.

Evenden, J.L. \& Ryan, C.N. (1996) The pharmacology of impulsive behaviour in rats: the effects of drugs on response choice with varying delays of reinforcement. Psychopharmacology (Berl.), 128, 161-170.

Florio, T., Capozzo, A., Cellini, R., Pizzuti, G., Staderini, E.M. \& Scarnati, E. (2001) Unilateral lesions of the pedunculopontine nucleus do not alleviate subthalamic nucleus-mediated anticipatory responding in a delayed sensorimotor task in the rat. Behav. Brain Res., 126, 93-103.

Funkiewiez, A., Ardouin, C., Krack, P., Fraix, V., Van Blercom, N., Xie, J., Moro, E., Benabid, A.L. \& Pollak, P. (2003) Acute psychotropic effects of bilateral subthalamic nucleus stimulation and levodopa in Parkinson's disease. Mov. Disord., 18, 524-530.

van Gaalen, M.M., Brueggeman, R.J., Bronius, P.F., Schoffelmeer, A.N. \& Vanderschuren, L.J. (2006a) Behavioral disinhibition requires dopamine receptor activation. Psychopharmacology (Berl.), 187, 73-85.

van Gaalen, M.M., van Koten, R., Schoffelmeer, A.N. \& Vanderschuren, L.J. (2006b) Critical involvement of dopaminergic neurotransmission in impulsive decision making. Biol. Psychiat., 60, 66-73.

Garcia, L., D'Alessandro, G., Bioulac, B. \& Hammond, C. (2005) Highfrequency stimulation in Parkinson's disease: more or less? Trends Neurosci., 28, 209-216.

Gueorguieva, R. \& Krystal, J.H. (2004) Move over ANOvA: progress in analyzing repeated-measures data and its reflection in papers published in the Archives of General Psychiatry. Arch. Gen. Psychiat., 61, 310-317.

Hershey, T., Revilla, F.J., Wernle, A., Gibson, P.S., Dowling, J.L. \& Perlmutter, J.S. (2004) Stimulation of STN impairs aspects of cognitive control in PD. Neurology, 62, 1110-1114.

Ho, M.Y., Wogar, M.A., Bradshaw, C.M. \& Szabadi, E. (1997) Choice between delayed reinforcers: interaction between delay and deprivation level. $Q . J$. Exp. Psychol. B, 50, 193-202.

Ho, M.Y., Mobini, S., Chiang, T.J., Bradshaw, C.M. \& Szabadi, E. (1999) Theory and method in the quantitative analysis of 'impulsive choice' behaviour: implications for psychopharmacology. Psychopharmacology (Berl.), 146, 362-372.

Holland, B.S. \& Copenhaver, M.D. (1988) Improved Bonferroni-type multiple testing procedures. Psychol. Bull., 104, 145-149.

Houeto, J.L., Mesnage, V., Mallet, L., Pillon, B., Gargiulo, M., du Moncel, S.T., Bonnet, A.M., Pidoux, B., Dormont, D., Cornu, P. \& Agid, Y. (2002) Behavioural disorders, Parkinson's disease and subthalamic stimulation. J. Neurol. Neurosurg. Psychiat., 72, 701-707.

Iversen, S.D. \& Mishkin, M. (1970) Perseverative interference in monkeys following selective lesions of the inferior prefrontal convexity. Exp. Brain Res., 11, 376-386.

Kita, H. \& Kitai, S.T. (1987) Efferent projections of the subthalamic nucleus in the rat: light and electron microscopic analysis with the PHA-L method. J. Comp. Neurol., 260, 435-452.

Lee, K.H., Blaha, C.D., Harris, B.T., Cooper, S., Hitti, F.L., Leiter, J.C., Roberts, D.W. \& Kim, U. (2006) Dopamine efflux in the rat striatum evoked by electrical stimulation of the subthalamic nucleus: potential mechanism of action in Parkinson's disease. Eur. J. Neurosci., 23, 1005-1014. 
Lewis, P. \& Dougherty, D.M. (1992) Pigeon performance on a variable-interval omission schedule at different levels of food deprivation. Behav. Process., 27, 27-36.

Lozano, A.M., Dostrovsky, J., Chen, R. \& Ashby, P. (2002) Deep brain stimulation for Parkinson's disease: disrupting the disruption. Lancet Neurol., 1, 225-231.

Maurice, N., Deniau, J.M., Glowinski, J. \& Thierry, A.M. (1998a) Relationships between the prefrontal cortex and the basal ganglia in the rat: physiology of the corticosubthalamic circuits. J. Neurosci., 18, 95399546.

Maurice, N., Deniau, J.M., Menetrey, A., Glowinski, J. \& Thierry, A.M. (1998b) Prefrontal cortex-basal ganglia circuits in the rat: involvement of ventral pallidum and subthalamic nucleus. Synapse, 29, 363-370.

Meissner, W., Reum, T., Paul, G., Harnack, D., Sohr, R., Morgenstern, R. \& Kupsch, A. (2001) Striatal dopaminergic metabolism is increased by deep brain stimulation of the subthalamic nucleus in 6-hydroxydopamine lesioned rats. Neurosci. Lett., 303, 165-168.

Monakow, K.H., Akert, K. \& Kunzle, H. (1978) Projections of the precentral motor cortex and other cortical areas of the frontal lobe to the subthalamic nucleus in the monkey. Exp. Brain Res., 33, 395-403.

O’Donnell, J.M. \& Seiden, L.S. (1983) Differential-reinforcement-of-low-rate 72-second schedule: selective effects of antidepressant drugs. J. Pharmacol. Exp. Ther, 224, 80-88.

Parent, A. \& Hazrati, L.N. (1995) Functional anatomy of the basal ganglia. II. The place of subthalamic nucleus and external pallidum in basal ganglia circuitry. Brain Res. Rev., 20, 128-154.

Phillips, J.M. \& Brown, V.J. (1999) Reaction time performance following unilateral striatal dopamine depletion and lesions of the subthalamic nucleus in the rat. Eur. J. Neurosci., 11, 1003-1010.

Pollard, G.T. \& Howard, J.L. (1986) Similar effects of antidepressant and nonantidepressant drugs on behavior under an interresponse-time greater than 72-s schedule. Psychopharmacology (Berl.), 89, 253-258.

Richards, J.B. \& Seiden, L.S. (1991) A quantitative interresponse-time analysis of DRL performance differentiates similar effects of the antidepressant desipramine and the novel anxiolytic gepirone. J. Exp. Anal. Behav., 56, 173-192.

Richards, J.B., Sabol, K.E. \& Seiden, L.S. (1993) DRL interresponse-time distributions: quantification by peak deviation analysis. J. Exp. Anal. Behav., 60, 361-385.

Robbins, T.W. (1996) Dissociating executive functions of the prefrontal cortex. Philos. Trans. R. Soc. Lond. B Biol. Sci., 351, 1463-1470.

Sabol, K.E., Richards, J.B., Layton, K. \& Seiden, L.S. (1995) Amphetamine analogs have differential effects on DRL 36-s schedule performance. Psychopharmacology (Berl.), 121, 57-65.

Sato, M. \& Hikosaka, O. (2002) Role of primate substantia nigra pars reticulata in reward-oriented saccadic eye movement. J. Neurosci., 22, 2363-2373.

Seiden, L.S., Andresen, J. \& MacPhail, R.C. (1979) Methylphenidate and d-amphetamine: effects and interactions with alphamethyltyrosine and tetrabenazine on DRL performance in rats. Pharmacol. Biochem. Behav. 10, $577-584$

Smith, I.D. \& Grace, A.A. (1992) Role of the subthalamic nucleus in the regulation of nigral dopamine neuron activity. Synapse, 12, 287-303.

Takeshita, S., Kurisu, K., Trop, L., Arita, K., Akimitsu, T. \& Verhoeff, N.P. (2005) Effect of subthalamic stimulation on mood state in Parkinson's disease: evaluation of previous facts and problems. Neurosurg. Rev., 28 , $179-186$.

Talpos, J.C., Wilkinson, L.S. \& Robbins, T.W. (2006) A comparison of multiple 5-HT receptors in two tasks measuring impulsivity. J. Psychopharmacol., 20, 47-58.

Tindell, A.J., Berridge, K.C., Zhang, J., Pecina, S. \& Aldridge, J.W. (2005) Ventral pallidal neurons code incentive motivation: amplification by mesolimbic sensitization and amphetamine. Eur. J. Neurosci., 22, 2617-2634.

Uslaner, J.M., Yang, P. \& Robinson, T.E. (2005) Subthalamic nucleus lesions enhance the psychomotor-activating, incentive motivational, and neurobiological effects of cocaine. J. Neurosci., 25, 8407-8415.

Wade, T.R., de Wit, H. \& Richards, J.B. (2000) Effects of dopaminergic drugs on delayed reward as a measure of impulsive behavior in rats. Psychopharmacology (Berl.), 150, 90-101.

Winstanley, C.A., Dalley, J.W., Theobald, D.E. \& Robbins, T.W. (2003) Global 5-HT depletion attenuates the ability of amphetamine to decrease impulsive choice on a delay discounting task in rats. Psychopharmacology (Berl.), 170, 320-331.

Winstanley, C.A., Dalley, J.W., Theobald, D.E. \& Robbins, T.W. (2004a) Fractionating impulsivity: contrasting effects of central 5-HT depletion on different measures of impulsive behavior. Neuropsychopharmacology, 29, 1331-1343.

Winstanley, C.A., Theobald, D.E., Cardinal, R.N. \& Robbins, T.W. (2004b) Contrasting roles of basolateral amygdala and orbitofrontal cortex in impulsive choice. J. Neurosci., 24, 4718-4722.

Winstanley, C.A., Baunez, C., Theobald, D.E. \& Robbins, T.W. (2005) Lesions to the subthalamic nucleus decrease impulsive choice but impair autoshaping in rats: the importance of the basal ganglia in Pavlovian conditioning and impulse control. Eur. J. Neurosci., 21, 3107-3116.

Witjas, T., Baunez, C., Henry, J.M., Delfini, M., Regis, J., Cherif, A.A., Peragut, J.C. \& Azulay, J.P. (2005) Addiction in Parkinson's disease: impact of subthalamic nucleus deep brain stimulation. Mov. Disord., 20, 1052-1055.

Wogar, M.A., Bradshaw, C.M. \& Szabadi, E. (1992) Choice between delayed reinforcers in an adjusting-delay schedule: the effects of absolute reinforcer size and deprivation level. Q. J. Exp. Psychol. B, 45, 1-13.

Wyvell, C.L. \& Berridge, K.C. (2000) Intra-accumbens amphetamine increases the conditioned incentive salience of sucrose reward: enhancement of reward 'wanting' without enhanced 'liking' or response reinforcement. J. Neurosci., 20, 8122-8130.

Wyvell, C.L. \& Berridge, K.C. (2001) Incentive sensitization by previous amphetamine exposure: increased cue-triggered 'wanting' for sucrose reward. J. Neurosci., 21, 7831-7840. 